\title{
Quasiperfect Domination in Trees
}

\author{
Jose Cáceres, Maria Luz Puertas ${ }^{1}$ \\ Estadística y Matemática Aplicada, Universidad de Almería, Almería, Spain \\ Carmen Hernando, Merce Mora, Ignacio M Pelayo ${ }^{2}$ \\ Matemàtica Aplicada, Universitat Politècnica de Catalunya, Barcelona, Spain
}

\begin{abstract}
A $k$-quasiperfect dominating set $(k \geq 1)$ of a graph $G$ is a vertex subset $S$ such that every vertex not in $S$ is adjacent to at least one and at most $k$ vertices in $S$. The cardinality of a minimum $k$-quasiperfect dominating set of $G$ is denoted by $\gamma_{1 k}(G)$. Those sets were first introduced by Chellali et al. (2013) as a generalization of the perfect domination concept (which coincides with the case $k=1$ ) and allow us to construct a decreasing chain of quasiperfect dominating parameters
\end{abstract}

$$
\gamma_{11}(G) \geq \gamma_{12}(G) \geq \ldots \geq \gamma_{1, \Delta}(G)=\gamma(G),
$$

in order to indicate how far is $G$ from being perfectly dominated. In this work, we study general properties, tight bounds, existence and realization results involving the parameters of the so-called $Q P$-chain (1), for trees.

Keywords: Domination, Perfect domination, Quasiperfect domination, Trees.

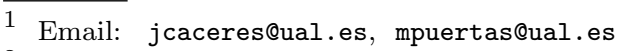

2 Email: carmen.hernando@upc.edu, merce.mora@upc.edu, ignacio.m.pelayo@upc.edu 


\section{Introduction}

Recall that a tree is a connected acyclic graph. A leaf is a vertex of degree 1 and vertices of degree at least 2 are interior vertices. We denote by $L(T)$ the set of leaves of a tree $T$ and by $\ell(T)$ the number of leaves of $T$. A support vertex is a vertex having at least a leaf in its neighborhood and a strong support vertex is a support vertex adjacent to at least two leaves.

Given a graph $G$, a subset $S$ of its vertices is a dominating set of $G$ if every vertex $v$ not in $S$ is adjacent to at least one vertex in $S$. The domination number $\gamma(G)$ is the minimum cardinality of a dominating set of $G$, and a dominating set of cardinality $\gamma(G)$ is called a $\gamma$-code [9].

An extreme way of domination occurs when every vertex not in $S$ is adjacent to exactly one vertex in $S$. In that case, $S$ is called a perfect dominating set [2] and $\gamma_{11}(G)$, the minimum cardinality of a perfect dominating set of $G$, is the perfect domination number. A dominating set of cardinality $\gamma_{11}(G)$ is called a $\gamma_{11}$-code.

In a perfect dominating set what is gained from the point of view of accuracy is lost in size, comparing it with a dominating set. Between both notions there is a graduation of definitions: $k$-quasiperfect domination. A $k$ quasiperfect dominating set for $k \geq 1$ ( $\gamma_{1 k}$-set for short) $[7,11]$ is a dominating set $S$ where every vertex not in $S$ is adjacent to at most $k$ vertices of $S$. Again the $k$-quasiperfect domination number $\gamma_{1 k}(G)$ is the minimum cardinality of a $\gamma_{1 k}$-set of $G$ and a $\gamma_{1 k}$-code is a $\gamma_{1 k}$-set of cardinality $\gamma_{1 k}(G)$.

Given a graph $G$ of order $n$ and maximum degree $\Delta, \gamma_{1 \Delta}$-sets are precisely dominating sets. Thus, one can construct the following chain of quasiperfect domination parameters:

$$
n \geq \gamma_{11}(G) \geq \gamma_{12}(G) \geq \ldots \geq \gamma_{1 \Delta}(G)=\gamma(G),
$$

known as the quasiperfect chain of $G$, or simply the $Q P$-chain of $G$.

\section{Known general results}

In this section, we review some results founded in the literature about quasiperfect parameters. Table 2 summarizes the values of parameters under consideration for some simple families of graphs.

Theorem 2.1 [7] If $G$ is a graph of order $n$ verifying at least one of the following conditions: (1) $\Delta(G) \geq n-3$; (2) $\Delta(G) \leq 2$; (3) $G$ is a cograph; (4) $G$ is a claw-free graph, then $\gamma_{12}(G)=\gamma(G)$. 


\begin{tabular}{ccccccc} 
& paths & cycles & cliques & stars & bicliques & wheels \\
\hline$G$ & $P_{n}$ & $C_{n}$ & $K_{n}$ & $K_{1, n-1}$ & $K_{p, n-p}$ & $W_{n}$ \\
\hline$n$ & $n \geq 3$ & $n \geq 4$ & $n \geq 2$ & $n \geq 4$ & $2 \leq p \leq n-p$ & $n \geq 3$ \\
\hline$\Delta(G)$ & 2 & 2 & $n-1$ & $n-1$ & $n-p$ & $n-1$ \\
\hline$\gamma_{11}(G)$ & $\left\lceil\frac{n}{3}\right\rceil$ & $\left\lceil\frac{2 n}{3}\right\rceil-\left\lfloor\frac{n}{3}\right\rfloor$ & 1 & 1 & 2 & 1 \\
$\gamma_{12}(G)$ & $\left\lceil\frac{n}{3}\right\rceil$ & $\left\lceil\frac{n}{3}\right\rceil$ & 1 & 1 & 2 & 1 \\
$\gamma(G)$ & $\left\lceil\frac{n}{3}\right\rceil$ & $\left\lceil\frac{n}{3}\right\rceil$ & 1 & 1 & 2 & 1 \\
\hline
\end{tabular}

Proposition 2.2 [3] Let $G=(V, E)$ a graph of order $n$.

(i) If $\gamma(G) \leq \Delta(G)$, then $\gamma_{1 \gamma}(G)=\ldots=\gamma_{1 \Delta}(G)=\gamma(G)$;

(ii) $\gamma_{1 \delta}(G)<n$;

(iii) $\gamma_{11}(G)=1$ if and only if $\Delta(G)=n-1$.

(iv) $\gamma_{11}(G) \leq n-\ell(G)$ where $\ell(G)$ is the number of vertices of degree one.

Theorem 2.3 [3] Let $k, n$ be positive integers such that $n \geq 6$ and $2 \leq k \leq n$. Then, there exists a graph $G$ of order $n$ such that $\Delta(G)=n-2$ and $\gamma_{11}(G)=k$.

Theorem 2.4 [3] Let $(h, k, n)$ be a triple of integers such that $2 \leq h \leq 3$, $2 \leq k \leq n$ and $n \geq 9$. Then, there exists a graph $G$ such that $|V(G)|=n$, $\Delta(G)=n-3, \gamma(G)=h$ and $\gamma_{11}(G)=k$.

Theorem 2.5 [3] Let $G$ be a graph of order $n$ and $\Delta(G)=3$, other than the bull graph. Then, $\gamma_{11}(G) \leq n-3$.

Proposition 2.6 [3] Let $G$ be either a cubic graph other than $K_{4}$, or a tree with order $n \geq 7$ and $\Delta(G)=3$. Then, $\gamma_{11}(G) \leq n-4$.

The join $G=G_{1} \vee G_{2}$ of graphs $G_{1}$ and $G_{2}$ is the graph such that $V(G)=$ $V\left(G_{1}\right) \cup V\left(G_{2}\right)$ and $E(G)=E\left(G_{1}\right) \cup E\left(G_{2}\right) \cup\left\{u v: u \in V\left(G_{1}\right), v \in V\left(G_{2}\right)\right\}$.

Theorem 2.7 [3] Let $G=G_{1} \vee G_{2}$ be a join graph of order $n$. Then,

(i) $\gamma_{11}(G)=1$ if and only if $G_{1}$ or $G_{2}$ have a universal vertex.

(ii) $\gamma_{11}(G)=2$ if and only if both $G_{1}$ and $G_{2}$ have at least an isolated vertex.

(iii) $\gamma_{11}(G)=n$ in other case. 
Corollary 2.8 [3] Let $G=G_{1} \vee G_{2}$ be a connected cograph without universal vertices. Then, $\gamma_{11}(G)=2$ if both $G_{1}$ and $G_{2}$ have at least an isolated vertex, and $\gamma_{11}(G)=n$ in any other case.

Theorem 2.9 [3] Let $h, k, n$ be integers such that $4 \leq n, 2 \leq h \leq k \leq n$ satisfying either $h+k \leq n$ or $3 h+k+1 \leq 2 n$. Then, there exists a claw-free graph $G$ of order $n$ such that $\gamma(G)=h$ and $\gamma_{11}(G)=k$.

The corona of a graph $G$, denoted by $\operatorname{cor}(G)$, is the graph obtained by attaching a leaf to each vertex of $G$.

Theorem 2.10 [8,10] For any graph $G$ the domination number satisfies $\gamma(G) \leq$ $n / 2$. And if $G$ is a graph of even order $n$, then $\gamma(G)=n / 2$ if and only if $G$ is the cycle of order 4 or the corona of a connected graph.

Graphs with odd order $n$ and maximum domination number $\gamma(G)=\lfloor n / 2\rfloor$ are also completely characterized in [1], as a list of six graph classes.

Proposition 2.11 [5] Let $T$ be a tree of order $n \geq 3$. Then

(i) Every $\gamma$-code of $T$ contains all its strong support vertices.

(ii) Every $\gamma_{11}$ - code of $T$ contains all its strong support vertices.

(iii) $\gamma_{11}(T) \leq n / 2$.

(iv) $\gamma_{11}(T)=n / 2$ if and only if $\gamma(T)=n / 2$ if and only if $T=\operatorname{cor}\left(T^{\prime}\right)$ for some tree $T^{\prime}$.

A tree for which removal of all its leaves results in a path is called a caterpillar.

Proposition 2.12 [7] If $T$ is a caterpillar, then $\gamma(T)=\gamma_{12}(T)$.

\section{Our results on Trees}

Theorem 3.1 [4] Let $T$ be a tree. Then, $\gamma_{1 k}(T) \leq \gamma(T)+\left\lceil\frac{\gamma(T)}{k}\right\rceil-1$, for every integer $k \in\{1, \ldots, \Delta(T)\}$.

Corollary 3.2 For every tree $T, \gamma_{11}(T) \leq 2 \gamma(T)-1$.

Remark 3.3 This bound is not true for general graphs and the difference between both parameters can be as large as desired. For example, the graph displayed in Figure 1 satisfies $\gamma(G)=2$ and $\gamma_{11}(G)=|V(G)|>2 \gamma(G)-1$.

Next, we present a realization theorem for the short chain $\gamma \leq \gamma_{11}(T) \leq$ $2 \gamma-1$. Note that, for every caterpillar $T$ of order $n \geq 3$, Proposition 2.12 


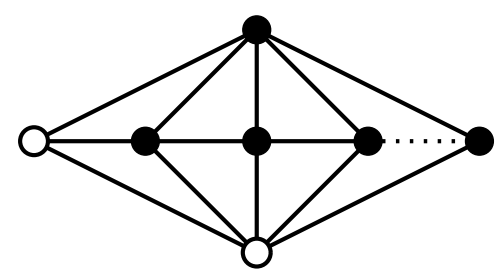

Fig. 1. The pair of white vertices form a $\gamma$-code.

and Corollary 3.2 just allow two possible situations, namely, either $\gamma(T)=$ $\gamma_{11}(T) \leq n / 2$ or $\gamma(T)<\gamma_{11}(T)<n / 2$. In the following result, we show that both of them are feasible and that parameters $\gamma$ and $\gamma_{11}$ can take every possible value in each case.

Proposition 3.4 [4] Let $a, b, n$ be positive integers.

(i) If $2 \leq 2 a \leq n$, then there exists a caterpillar $T$ of order $n$ such that $\gamma(T)=\gamma_{11}(T)=a$.

(ii) If $2 \leq a<b \leq 2 a-1$ and $n>2 b$, then there exists a caterpillar $T$ of order $n$ such that $\gamma(T)=a$ and $\gamma_{11}(T)=b$.

Proposition 3.5 [4] A caterpillar $T$ satisfies $\gamma_{11}(T)=2 \gamma(T)-1$ if and only if belongs to the family shown in Figure 2.

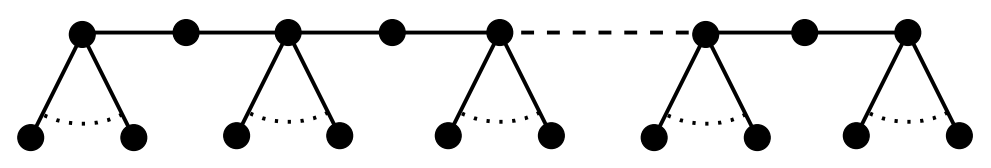

Fig. 2. Caterpillar with $\gamma_{11}(T)=2 \gamma(T)-1$.

Let $T$ a tree with maximum degree $\Delta \geq 3$. Next theorem shows that for each inequality of the QP-chain, both possibilities, the equality and the strict inequality, are feasible.

Theorem 3.6 [4] There exists a tree with maximum degree $\Delta \geq 3$, satisfying each one of the $2^{\Delta-1}$ possible combinations of the inequalities of the QP-chain.

Finally, we present the general form of the QP-chain in the case of $k$-ary trees, that has just two different terms.

Proposition 3.7 [4] Let $T=T(k, h)$ the full $k$-ary tree of order $n=\frac{k^{h+1}-1}{k-1}$, where all leaves are at distance $h-1$ from the root, with $k \geq 2, h \geq 3$. Then $n-\ell(T)=\gamma_{11}(T)=\gamma_{12}(T)=\ldots=\gamma_{1, k-1}(T)>\gamma_{1, k}(T)=\gamma_{1, k+1}(T)=\gamma(T)$ 


\section{References}

[1] X. Baogen, E.J. Cockayne, T.W. Haynes, S.T. Hedetniemi and Z. SHANGCHAO: Extremal graphs for inequalities involving domination parameters. Discrete Math., 216 (2000), 1-10.

[2] N. Biggs: Perfect codes in graphs. J. Combin. Theory Ser. B, 15 (1973), 289296.

[3] J. Cáceres, C. Hernando, M. Mora, I. M. Pelayo and M. L. Puertas: On Perfect and Quasiperfect Dominations in Graphs. Submitted. http://arxiv.org/pdf/1411.7818v1.pdf

[4] J. Cáceres, C. Hernando, M. Mora, I. M. Pelayo and M. L. Puertas: Perfect and Quasiperfect Dominations in Trees. Submitted.

[5] Y. Caro, A. Hansberg and M. Henning: Fair domination in graphs. Discrete Math., 312 (19) (2012), 2905-2914.

[6] G. Chartrand, L. Lesniak, P. Zhang: Graphs and Digraphs, (5th edition). CRC Press, Boca Raton, Florida, 2011.

[7] M. Chellali, T.W. Haynes, S.T. Hedetniemi, A. McRae: [1, 2]-sets in graphs. Discrete Appl. Math., 161(18) (2013), 2885-2893.

[8] J.F. Fink, M.S. Jacobson, L.F. Kinch And J. Roberts: On graphs having domination number half their order. Periodica Mathematica Hungarica, 16 (1985), 287-293.

[9] T.W. Haynes, S.T. Hedetniemi, P.J. Slater: Fundamentals of domination in graphs. Marcel Dekker, New York, 1998.

[10] C. Payan and N. H. Xuong: Domination-balanced graphs. J. Graph Theory, 6 (1982), 23-32.

[11] X. YAng, B. Wu. [1,2]-domination in graphs. Discrete Appl. Math. 175 (2014), $79-86$. 


\section{Appendix}

\section{Proof of Theorem 3.1}

Remark 1 Let $T$ be a tree and $S$ a dominating set. Then, since $T$ has no cyles, every vertex not in $S$ has at most one neighbor at each connected component of the subgraph $T[S]$.

Remark 2 Let $T$ be a tree and $S$ a dominating set such that the subgraph $T[S]$ has at most $k$ connected components. Then, $S$ is a $\gamma_{1 k}$-set.

Let $S$ be a $\gamma$-code of $T$. If $S$ is also a $\gamma_{1 k}$-set, then the inequality stated in the theorem holds.

Suppose on the contrary that $S$ is not a $\gamma_{1 k}$-set.

We construct a $\gamma_{1 k}$-set $S^{*}$ containing $S$ and satisfying the inequality stated in the theorem. Let $r$ be the number of connected components of the subgraph induced by $S$, denoted by $T[S]$. Then, $\gamma(T) \geq r$ and, by Remark $\mathbf{2}, r>k$.

Consider a vertex $x_{0} \in V(T) \backslash S$ with at least $k+1$ neighbors in $S$ and let $S_{1}=S \cup\left\{x_{0}\right\}$. By Remark 1, all the neighbors of $x_{0}$ in $S$ lie in different connected components of $T[S]$, therefore $S_{1}$ is a dominating set inducing a subgraph $T\left[S_{1}\right]$ with at most $r-k$ connected components. If $S_{1}$ is a $\gamma_{1 k}$-set, let $S^{*}=S_{1}$.

Otherwise, consider a vertex $x_{1} \in V(T) \backslash S_{1}$ having at least $k+1$ neighbors in $S_{1}$ and let $S_{2}=S_{1} \cup\left\{x_{1}\right\}$. By Remark 1, all the neighbors of $x_{1}$ in $S_{1}$ lie in different connected components of $T\left[S_{1}\right]$, therefore $S_{2}$ is a dominating set inducing a subgraph $T\left[S_{2}\right]$ with at most $(r-k)-k=r-2 k$ connected components. If $S_{2}$ is a $\gamma_{1 k}$-set, let $S^{*}=S_{2}$.

Otherwise, we repeat this procedure until we obtain a $\gamma_{1 k}$-set. Observe that this procedure will end since the number of connected components induced by the sets $S_{1}, S_{2}, \ldots$ is strictly decreasing. Moreover, since $T\left[S_{i}\right]$ has at most $r-i k$ connected components, by Remark $2, S_{i}$ is a $\gamma_{1 k}$-set whenever $r-i k \leq$

$k$. Therefore, the number of steps needed in order to obtain that $S_{i}$ is a $\gamma_{1 k}$-set, is at most $i=\left\lceil\frac{r-k}{k}\right\rceil$.

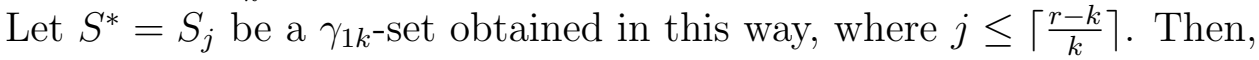

$\gamma_{1 k}(T) \leq\left|S^{*}\right|=|S|+j \leq \gamma(T)+\left\lceil\frac{r-k}{k}\right\rceil \leq \gamma(T)+\left\lceil\frac{\gamma(T)-k}{k}\right\rceil=\gamma(T)+\left\lceil\frac{\gamma(T)}{k}\right\rceil-1$. 


\section{Proof of Proposition 3.4}

(i) Consider the caterpillar obtained by attaching a leaf to each of the first $a-1$ vertices of a path of order $a$ and $n-2 a+1 \geq 1$ leaves to the last vertex of the path (see Figure 3). Then the vertices of the path is both a $\gamma$-code and a $\gamma_{11}$-code, and $\gamma(T)=\gamma_{11}(T)=a$.

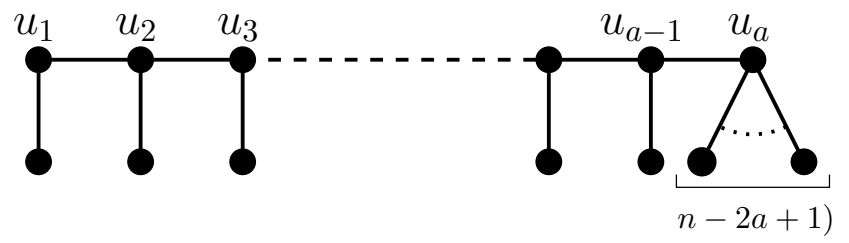

Fig. 3. $T$ has order $n, \gamma(T)=\gamma_{11}(T)=a$.

(ii) Note that $\gamma(T)=1$ implies $\gamma_{11}(T)=1$, so if both parameter do not agree them $\gamma(T) \geq 2$.

Using that $1 \leq b-a \leq a-1$, let $P$ be the path of order $b$ with consecutive vertices labeled with

$$
u_{1}, v_{1}, \ldots, u_{b-a}, v_{b-a}, u_{b-a+1}, u_{b-a+2}, \ldots, u_{a}
$$

and consider the caterpillar obtained by attaching two leaves to each of the vertices $u_{1}, u_{2}, \ldots, u_{b-a}$, one leaf to each of the vertices $u_{b-a+2}, u_{b-a+3}, \ldots, u_{a}$ and $n-2 b+1$ leaves to vertex $u_{b-a+1}$ (see Figure 4 ). Since $n-2 b+$ $1 \geq 2$ we obtain that $\left\{u_{1}, u_{2}, \ldots, u_{a}\right\}$ is a $\gamma$-code with $a$ vertices and $\left\{u_{1}, u_{2}, \ldots, u_{a}\right\} \cup\left\{v_{1}, \ldots, v_{b-a}\right\}$ is a $\gamma_{11}$-code with $b$ vertices.

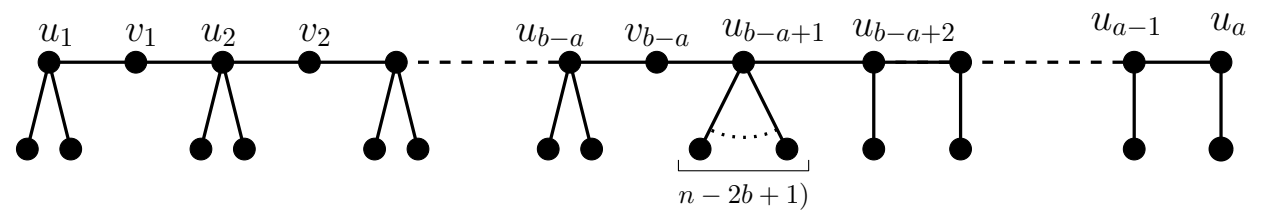

Fig. 4. $T$ has order $n>2 b, a=\gamma(T)<\gamma_{11}(T)=b \leq 2 a-1$. 


\section{Proof of Theorem 3.6}

Remark 1 If $u$ is a vertex of a graph $G$ with at least $d$ leaves in its neighborhood, then $u$ is in every $\gamma_{1, h}$-code, for any $h \in\{1, \ldots, d-1\}$.

Remark 2 If $G$ is a graph with maximum degree $\Delta$ and $u$ is a vertex with at least $\Delta-1$ leaves in its neighborhood, then $u$ is in every $\gamma_{1, h}$-code, for any $h \in\{1, \ldots, \Delta-2\}$.

Remark 3 Let $T$ be a tree with maximum degree $\Delta$ and $s$ support vertices. Then $\gamma_{1, \Delta}(T)=\gamma(T) \geq s$.

Let $\Delta \geq 3$. For all $i \in\{1, \ldots, \Delta-1\}$, we write $\circledast_{i}$ for the symbol ' $=$ ' or ' $>$ ' in $\gamma_{1, i}(T) \geq \gamma_{1, i+1}(T)$.

(i) Case 1. If $\circledast_{i}$ is ' $=$ ' for all $i \in\{1, \ldots, \Delta-2\}$. We distinguish two subcases.

(a) Case 1.1. If $\circledast_{\Delta-1}$ is ' $=$ '. The complete bipartite graph $T=K_{1, \Delta}$ is a tree with maximum degree $\Delta$ satisfying:

$$
\gamma_{11}(T)=\gamma_{12}(T)=\ldots=\gamma_{1, \Delta-1}(T)=\gamma_{1, \Delta}(T)=\gamma(T)=1 .
$$

(b) Case 1.2. If $\circledast_{\Delta-1}$ is ' $>$ '. We consider the following tree $T$ with maximum degree $\Delta$ : let $u$ be a vertex of degree $\Delta$ adjacent to vertices $x_{1}, x_{2}, \ldots, x_{\Delta}$, and attach $\Delta-1$ leaves to each $x_{i}, 1 \leq i \leq \Delta$. Then, we easily derive from Remark 2 that $\left\{x_{1}, \ldots, x_{\Delta}\right\}$ is a $\gamma$-code and $\left\{u, x_{1}, \ldots, x_{\Delta}\right\}$ is a $\gamma_{1, i}$-code for any $i$ such that $i<\Delta$. Therefore, $T$ satisfies

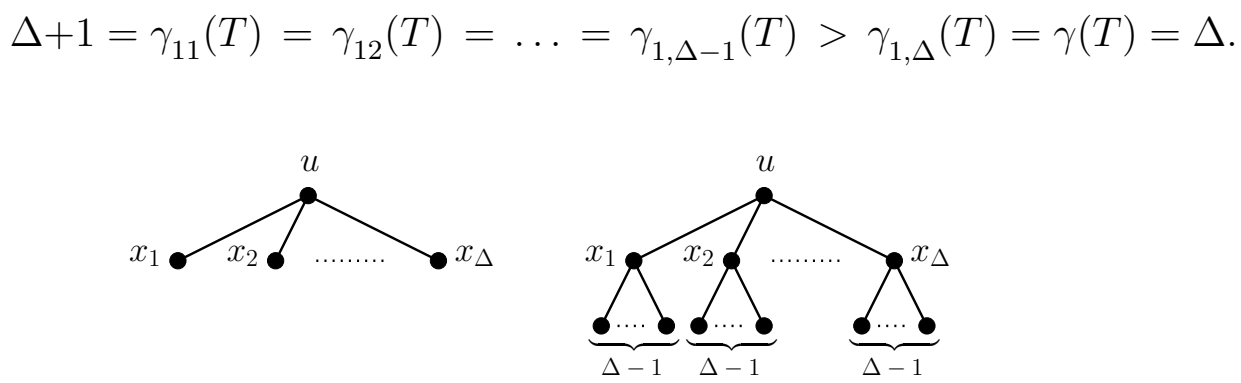
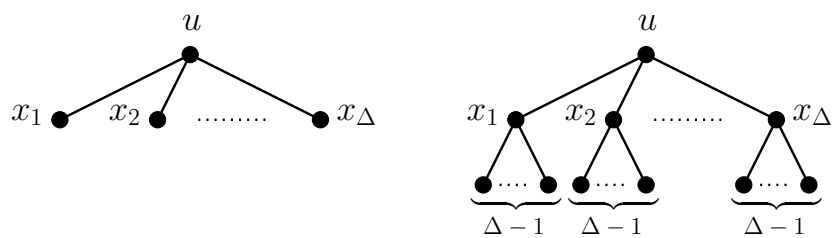

Fig. 5. Trees illustrating Case 1. of Theorem 3.6.

(ii) Case 2. If $\circledast_{i}$ is ' $>$ ' for some $i \in\{1, \ldots, \Delta-2\}$.

If $\Delta=3$, consider the graphs showed in Figure 6 . The tree $T$ on the left side satisfies $6=\gamma_{11}(T)>\gamma_{12}(T)=\gamma_{1,3}(T)=\gamma(T)=4$, since support vertices form a $\gamma$-code (and also a $\gamma_{12}$-code and a $\gamma_{13}$-code), and 
all vertices but the leaves form a $\gamma_{11}$-code. The tree $T$ on the right side satisfies $\gamma_{11}(T)=18>\gamma_{12}(T)=12>\gamma_{1,3}(T)=\gamma(T)=11$, since support vertices together with vertex $u$ form a $\gamma$-code (and also a $\gamma_{13^{-}}$ code), support vertices together with vertices $u$ and $v$ form a $\gamma_{12}$-code, and all vertices but the leaves form a $\gamma_{11}$-code.

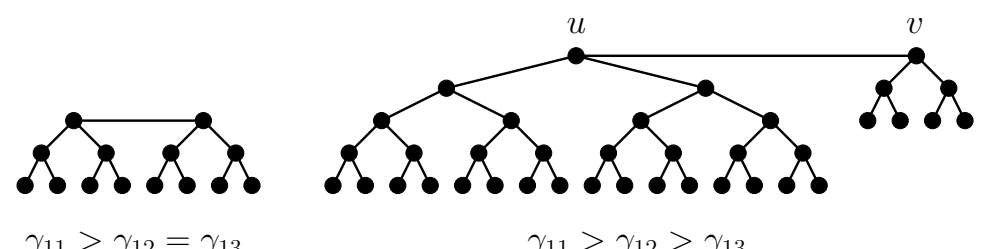

Fig. 6. Trees illustrating Case 2 of Theorem 3.6 when $\Delta=3$.

Now suppose $\Delta \geq 4$. Let

$$
\left\{i_{1}, i_{2}, \ldots, i_{k}\right\}=\left\{j: \gamma_{1, j}(T)>\gamma_{1, j+1}(T), j \leq \Delta-2\right\},
$$

where $k \geq 1$ by hypotheses, and assume $1 \leq i_{1}<\ldots<i_{k} \leq \Delta-2$. We distinguish two subcases.
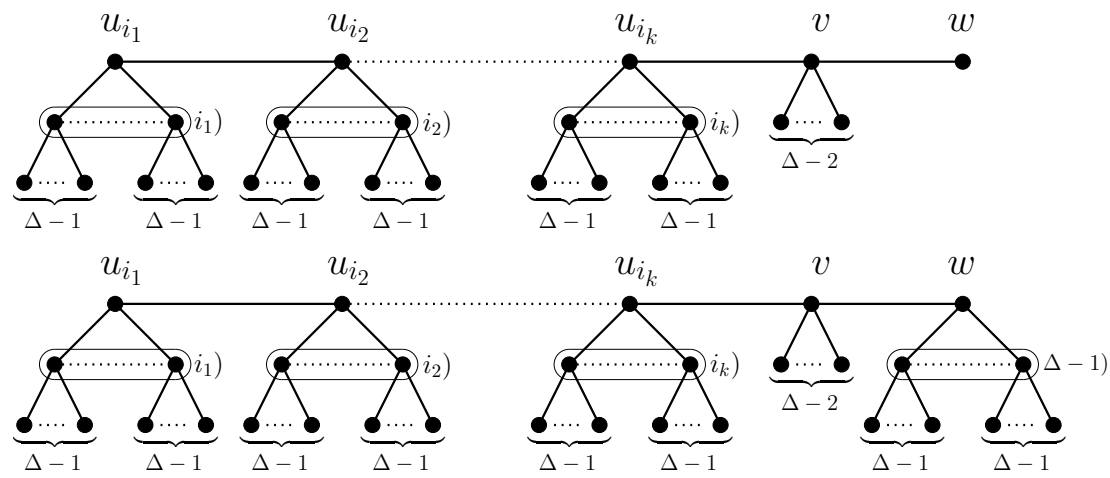

Fig. 7. Trees illustrating Case 2.1 (above) and Case 2.2 (bottom).

(a) Case 2.1. If $\circledast_{\Delta-1}$ is ' $=$ '.

Consider a path $P$ of length $k+2$ with consecutive vertices labeled $u_{i_{1}}, \ldots, u_{i_{k}}, v, w$. Attach $i_{j}$ new vertices to $u_{i_{j}}$ and $\Delta-1$ leaves to each one of those new vertices. Attach also $\Delta-2$ leaves to vertex $v$.

For each vertex $x$ of the path $P$, let $N^{\prime}(x)$ be the set of vertices of $N(x)$ not belonging to the path $P$. Let $A=\cup_{j=1}^{k} N^{\prime}\left(u_{i_{j}}\right)$.

It is not hard to verify that $A \cup\{v\}$ is a $\gamma$-code of $T$, and also a $\gamma_{1, \Delta-1}$-code. Moreover, $A \cup\{v\} \cup\left\{u_{i_{j}}: h \leq j \leq k\right\}$ is a $\gamma_{1 i}$-code if $i_{h-1}<i \leq i_{h}$. 
(b) Case 2.2. If $\circledast_{\Delta-1}$ is ' $>$ '.

Consider the tree constructed in case 2.1 and attach $\Delta-1$ new vertices to $w$ and $\Delta-1$ leaves to each one of those new vertices.

With the same notations as in Case 2.1, it is easy to verify that $A \cup\{v\} \cup N^{\prime}(w)$ is a $\gamma$-code of $T$ and $A \cup\{v, w\} \cup N^{\prime}(w)$ is a $\gamma_{1, \Delta-1^{-}}$ code. Moreover, $A \cup\{v, w\} \cup N^{\prime}(w) \cup\left\{u_{i_{j}}: h \leq j \leq k\right\}$ is a $\gamma_{1 i}$-code if $i_{h-1}<i \leq i_{h}$.

Lemma 3.8 Let $T$ be a tree of order $n \geq k+1(k \geq 2)$ with all interior vertices of degree at least $k+1$, except at most one vertex of degree $k$, then $\gamma_{1, k-1}(T)=n-\ell(T)$.

Proof. Notice that $V(T) \backslash L(T)$ is a $\gamma_{1, k-1}$-set for all $k \geq 2$. Suppose that $S$ is a $\gamma_{1, k-1}$-code such that $S \neq V(T) \backslash L(T)$. If $V(T) \backslash L(T) \subset S$, then $|S|>|V(T) \backslash L(T)|$ which is a contradiction. Therefore, there exists a vertex $u_{0} \in V(T) \backslash L(T)$ such that $u_{0} \notin S$. Consider the connected component $T_{0}$ of $u_{0}$ in $T \backslash S$. Notice that $T_{0}$ is a tree of order $n_{0} \geq 1$. If $T_{0}$ has only the vertex $u_{0} \notin L(T)$, then $u_{0}$ is adjacent to at least $k$ vertices of $S$, which is a contradiction. If $T_{0}$ has at least two vertices, $T_{0}$ has at least two leaves in $T_{0}$. Observe that a leaf $w$ of $T_{0}$ can not be a leaf of $T$, otherwise the only neighbor of $w$ is not in $S$, contradicting the fact that $S$ is a dominating set. Therefore, $T_{0}$ has a leaf $w_{0}$ that is a vertex of degree al least $k+1$, implying that $\geq k$ neighbors of $w_{0}$ are in $S$, which is again a contradiction.

\section{Proof of Proposition 3.7}

The set of interior vertices of a tree is a $\gamma_{1, i}$-set for any $i \geq 1$. Therefore, by Lemma 3.8, $n-\ell(T)=\gamma_{11}(T)=\gamma_{12}(T)=\ldots=\gamma_{1, k-1}(T)$. On the other hand, for any $h \geq 3$ consider the set $S$ described as follows:

$$
\begin{aligned}
& S=\bigcup_{0 \leq i \leq r-1} L_{2+3 i}, \text { if } h=3 r, r \geq 1 ; \\
& S=\{z\} \cup \bigcup_{1 \leq i \leq r} L_{3 i}, \text { where } z \in L_{2} \text {, if } h=3 r+1, r \geq 1 ; \\
& S=\bigcup_{0 \leq i \leq r} L_{1+3 i}, \text { if } h=3 r+2, r \geq 1 .
\end{aligned}
$$

Notice that $S$ contains exactly the vertices of one of each three consecutive levels, taking into account that $S$ must contain the strong support vertices, i.e., the vertices of level $h-1$, and in the case $h=3 r+1$ we have to add a vertex $z$ of level 2 to dominate the root (see in Figure 8 an illustration of case $k=2)$. 


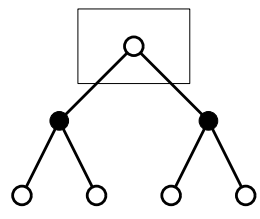

$\ldots$

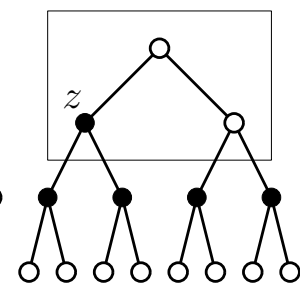

$\cdots \cdots$

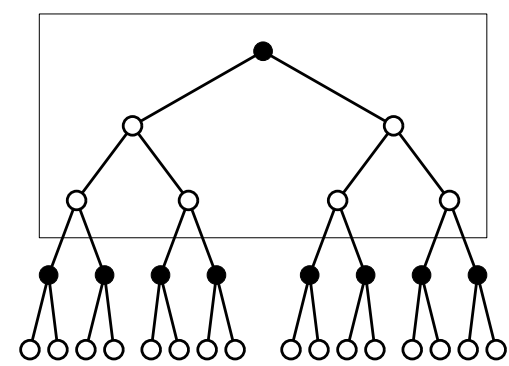

$$
h=3 r \quad h=3 r+1 \quad h=3 r+2
$$

Fig. 8. If we add new groups of three levels in each case, being black vertices those of the middle level, the set of black vertices is a dominating code of $T(2, h), h \geq 3$.

By construction, it is obvious that $S$ is a $\gamma_{1, k}$-set and a $\gamma_{1, k+1}$-set, since a vertex not in $S$ has at most $k$ neighbors in $S$. We claim that $S$ is a dominating code and consequently a $\gamma_{1, k}$-code and a $\gamma_{1, k+1}$-code. Let $S$ be a dominating code of $T(k, h), k \geq 2, h \geq 3$. We know that $S$ contains all its strong support vertices, $L_{h-1}$, and these vertices dominate vertices of levels $h, h-1$ and $h-2$. So, we may assume that $S$ does not contain any vertex of level $h-2$, otherwise we can change a vertex $x \in S \cap L_{h-2}$ by its neighbor in level $h-3$ obtaining also a dominating code. Therefore, $S$ is obtained by adding a dominating code of the tree $T(k, h-3)$. Reasoning recursively, we deduce that $S$ is a dominating code. 